\title{
Pricing and Hedging \\ for Incomplete Jump Diffusion Benchmark Models
}

\author{
Eckhard Platen ${ }^{1}$
}

October 7, 2003

\begin{abstract}
This paper considers a class of incomplete financial market models with security price processes that exhibit intensity based jumps. The benchmark or numeraire is chosen to be the growth optimal portfolio. Portfolio values, when expressed in units of the benchmark, are local martingales. In general, an equivalent risk neutral martingale measure need not exist in the proposed framework. Benchmarked fair derivative prices are defined as conditional expectations of future benchmarked prices under the real world probability measure. This concept of fair pricing generalizes classical risk neutral pricing. The pricing under incompleteness is modeled by the choice of the market prices for risk. The hedging is performed under minimization of profit and loss fluctuations.
\end{abstract}

1991 Mathematics Subject Classification: primary 90A12; secondary 60G30, 62P20. primary 90A12; secondary 60G30, 62P20.

JEL Classification: G10, G13

Key words and phrases: benchmark model, jump diffusions, incomplete market, growth optimal portfolio, fair pricing, hedge error minimization.

\footnotetext{
${ }^{1}$ University of Technology Sydney, School of Finance \& Economics and Department of Mathematical Sciences, PO Box 123, Broadway, NSW, 2007, Australia
} 


\section{Introduction}

In the case of complete markets without jumps Long (1990) and Bajeux-Besnainou \& Portait (1997) have proposed the use of the growth optimal portfolio (GOP) as numeraire, where the corresponding pricing measure is the real world probability measure. Along these lines in Platen (2002, 2004) pricing and hedging are performed for complete markets and jump diffusions without measure transformation. Geman, El Karoui \& Rochet (1995) describe how to use a tradable numeraire for pricing when a corresponding equivalent local martingale measure exists. Under such a condition Föllmer \& Sondermann (1986), Föllmer \& Schweizer (1991), Hofmann, Platen \& Schweizer (1992) and Heath, Platen \& Schweizer (2001b), have used for the pricing and hedging in incomplete markets the concept of local risk minimization, which results in the identification of a minimal equivalent martingale measure. Musiela \& Zariphopoulou (2003) have described an interesting utility maximization approach for valuing contingent claims in incomplete markets. El Karoui (2003) provides an elegant pricing methodology for utility based valuation criteria. In Elliott (2003) a duality based valuation method is described. For a recent survey on valuation, hedging and investing in incomplete markets we refer to Davis (2003).

This paper is based on the use of the GOP as benchmark or numeraire and establishes a consistent pricing and hedging concept in a class of incomplete market models with asset prices that follow jump diffusions. By avoiding the assumption on the existence of an equivalent local martingale measure one gains access to a wider class of models than would otherwise be the case using standard approaches. The freedom gained is important from the practical point of view. This is because certain realistic model classes, described in Platen (2001, 2002, 2004), cannot be covered by classical valuation approaches since for these models an equivalent pricing measure does not exist.

For contingent claim valuation the concept of a fair value process is introduced. Fair values, when expressed in units of the GOP, are martingales under the real world probability measure and are shown to coincide with the minimal replicating hedge portfolio in complete markets. The benchmarked fair price can be computed as the conditional expectation of future benchmarked prices. Fair pricing is a natural generalization of standard risk neutral pricing.

The proposed benchmark approach covers incomplete markets with securities that are not tradable. The paper emphasizes the fact that the choice of the market prices for risk determines the GOP and thus fair prices. As already indicated above, many different methods are known for valuing contingent claims in incomplete markets. They generally result in a specific choice of the market price for risk. This paper assumes that the market price for risk has been already modeled. It proposes to identify the hedging strategy by minimization of the fluctuations of the profit and loss process, which is called fluctuation minimization. This leads to a natural hedging strategy. 
This paper introduces in Section 2 a class of incomplete benchmark models with jumps. Pricing and hedging are studied in Section 3.

\section{Incomplete Benchmark Model with Jumps}

\subsection{Modeling Uncertainty}

In a given market the continuous uncertainty, for instance the trading noise, is modeled by $m$ independent standard Wiener processes $W^{k}=\left\{W^{k}(t), t \in[0, T]\right\}$, $k \in\{1,2, \ldots, m\}, m \in\{1,2, \ldots, d\}, d \in\{1,2, \ldots\}$. These are defined on a filtered probability space $\left(\Omega, \mathcal{A}_{T}, \underline{\mathcal{A}}, P\right)$ with finite time horizon $T \in(0, \infty)$. Event driven uncertainty, for instance the unexpected default of a company, is modeled by $d-m$ counting processes $p^{m+1}, \ldots, p^{d}$. Events of the $k$ th type are counted by the $\underline{\mathcal{A}}$-adapted, $k$ th counting process $p^{k}=\left\{p^{k}(t), t \in[0, T]\right\}$. The corresponding intensity $h^{k}=\left\{h_{t}^{k}, t \in[0, T]\right\}$ is a predictable, strictly positive process with

$$
h_{t}^{k}>0
$$

for $t \in[0, T]$ and $k \in\{m+1, \ldots, d\}$. The $k$ th jump martingale $W^{k}=\left\{W^{k}(t), t \in\right.$ $[0, T]\}$ is defined using the stochastic differential

$$
d W^{k}(t)=\left(h_{t}^{k}\right)^{-\frac{1}{2}}\left(d p^{k}(t)-h_{t}^{k} d t\right)
$$

for $k \in\{m+1, \ldots, d\}$ and $t \in[0, T]$. The jump martingales $W^{m+1}, \ldots, W^{d}$ are assumed not to jump at the same time.

We denote by $A^{\top}$ the transpose of a vector or matrix $A$. The evolution of market uncertainty is therefore modeled by the vector of independent $(\underline{\mathcal{A}}, P)$-martingales $W=\left\{W(t)=\left(W^{1}(t), \ldots, W^{d}(t)\right)^{\top}, t \in[0, T]\right\}$. Here the continuous martingales $W^{1}, \ldots, W^{m}$ are Wiener processes, whereas the jump martingales $W^{m+1}, \ldots$, $W^{d}$ are compensated and normalized counting processes. The filtration $\underline{\mathcal{A}}=$ $\left(\mathcal{A}_{t}\right)_{t \in[0, T]}$ is taken to be the augmentation under $P$ of the natural filtration $\mathcal{A}^{W}$, generated by the vector process $W$ and fulfilling the usual conditions with $\mathcal{A}_{0}$ as trivial $\sigma$-algebra, see Protter (1990). The increments $W^{k}(t+\varepsilon)-W^{k}(t)$ are assumed to be independent of $\mathcal{A}_{t}$ for all $t \in[0, T], \varepsilon>0$ and $k \in\{1,2, \ldots, d\}$.

\section{$2.2 \quad$ Primary Securities}

We introduce one riskless and $d$ risky primary security accounts with values $S^{(j)}(t)$ at time $t \in[0, T]$ for $j \in\{0,1, \ldots, d\}$. A primary security account is an investment account, consisting only of units of this type of security with all proceeds reinvested. Such account holds units in the corresponding primary security, for instance shares, as well as accrued income such as dividends or interest. A primary security account may describe the value process of a security that is not 
traded. In this case, values may be determined by modeling demand and supply or applying other valuation rules. Such a valuation rule can be given, for instance, by some utility maximization criterion. Since not all primary security accounts are traded, some cannot be used for hedging purposes. This makes the market in a practical sense incomplete. Note however that for all primary security accounts the dynamics are uniquely determined.

To be specific, we assume that the $j$ th primary security account value $S^{(j)}(t)$ at time $t$ is nonnegative and satisfies the Itô stochastic differential equation (SDE)

$$
d S^{(j)}(t)=S^{(j)}(t-)\left(a^{j}(t) d t+\sum_{k=1}^{d} b^{j, k}(t) d W^{k}(t)\right)
$$

for $t \in[0, T]$ with initial value $S^{(j)}(0)>0, j \in\{0,1, \ldots, d\}$, see Protter (1990). The 0th primary security account $S^{(0)}=\left\{S^{(0)}(t), t \in[0, T]\right\}$ denotes the savings account that continuously accrues interest according to the predictable short term interest rate process $r=\{r(t), t \in[0, T]\}$. For the savings account $S^{(0)}(t)$ we obtain from $(2.3)$

$$
a^{0}(t)=r(t)
$$

and

$$
b^{0, k}(t)=0
$$

for all $t \in[0, T]$ and $k \in\{1,2, \ldots, d\}$. Here the processes $a^{j}, b^{j, k}, r$ and $h^{k}$ are predictable and such that a strong, unique solution of the system of SDEs (2.3) exists, see Protter (1990). Therefore, the unique vector process $S=\{S(t)=$ $\left.\left(S^{(0)}(t), \ldots, S^{(d)}(t)\right)^{\top}, t \in[0, T]\right\}$ determines the evolution of the primary security accounts.

\subsection{Portfolios}

In this setting portfolios are formed as weighted combinations of primary security accounts. All value and wealth processes are assumed to be portfolios, which are characterized by strategies. A predictable stochastic process $\delta=\{\delta(t)=$ $\left.\left(\delta^{(0)}(t), \ldots, \delta^{(d)}(t)\right)^{\top}, t \in[0, T]\right\}$ is called a strategy if $\delta$ is $S$-integrable, see Protter (1990). The $j$ th component $\delta^{(j)}(t)$ of the strategy $\delta$ denotes the number of units of the $j$ th primary security account, held at time $t \in[0, T]$ in the corresponding portfolio, $j \in\{0,1, \ldots, d\}$. For a strategy $\delta$ we denote by $S^{(\delta)}(t)$ the value of the corresponding portfolio at time $t$, that is

$$
S^{(\delta)}(t)=\delta(t)^{\top} S(t)
$$

for $t \in[0, T]$. To quantify the notion of conservation of value within a portfolio we introduce the following definition. 
Definition 2.1 A strategy $\delta$ and corresponding portfolio process $S^{(\delta)}=\left\{S^{(\delta)}(t)\right.$, $t \in[0, T]\}$ are called self-financing if

$$
d S^{(\delta)}(t)=\sum_{j=0}^{d} \delta^{j}(t) d S^{(j)}(t)
$$

for all $t \in[0, T]$.

In the remaining part of this paper we will only consider self-financing strategies and the corresponding self-financing portfolios. Therefore, we omit from now on the word "self-financing".

It makes sense to consider only models, where one cannot generate from zero initial capital some strictly positive wealth. Otherwise, if this were not the case, then one would have a form of basic arbitrage. Let us give a more precise definition of such a notion.

Definition 2.2 A nonnegative portfolio process $S^{(\delta)}$ provides basic arbitrage if there exist stopping times $\tau$ and $\sigma$ with $0 \leq \tau<\sigma \leq T$ such that

$$
S^{(\delta)}(\tau)=0
$$

and

$$
P\left(S^{(\delta)}(\sigma)>0 \mid \mathcal{A}_{\tau}\right)>0
$$

almost surely.

Precluding basic arbitrage means that one cannot generate by using a nonnegative portfolio process strictly positive terminal wealth when starting from nothing. In the literature there exist many arbitrage definitions. For instance, the fundamental theorem of asset pricing in Delbaen \& Schachermayer $(1995,1998)$ provides an important link between the existence of an equivalent risk neutral measure and the, so called, no free lunch with vanishing risk no-arbitrage condition.

One way that basic arbitrage can arise is to have portfolio processes with the same martingale terms in their SDEs but with different drift terms. To exclude such kind of arbitrage opportunities the following natural assumption is made.

Assumption 2.3 We assume that the generalized volatility matrix $b(t)=$ $\left[b^{j, k}(t)\right]_{j, k=1}^{d}$ is for Lebesgue-almost-every $t \in[0, T]$ invertible.

This allows us to introduce the market price for risk vector

$$
\theta(t)=\left(\theta^{1}(t), \ldots, \theta^{d}(t)\right)^{\top}=b^{-1}(t)[a(t)-r(t) \mathbf{1}]
$$


with appreciation rate vector $a(t)=\left(a^{1}(t), \ldots, a^{d}(t)\right)^{\top}$ and unit vector $\mathbf{1}=(1, \ldots$, $1)^{\top}$ for $t \in[0, T]$. Without loss of generality, by using (2.10) we can rewrite the $\operatorname{SDE}(2.3)$ in the form

$$
d S^{(j)}(t)=S^{(j)}(t-)\left(r(t) d t+\sum_{k=1}^{d} b^{j, k}(t)\left(\theta^{k}(t) d t+d W^{k}(t)\right)\right)
$$

for $t \in[0, T]$ and $j \in\{0,1, \ldots, d\}$. For $k \in\{1,2, \ldots, m\}$ the quantity $\theta^{k}(t)$ is the market price for risk at time $t$ with respect to the $k$ th Wiener process $W^{k}$, see Karatzas \& Shreve (1998). Additionally, we have for $k \in\{m+1, \ldots, d\}$ another type of market price for risk, which can be interpreted as the market price for $k$ th event risk. Note that besides generalized volatilities $b^{j, k}, j, k \in\{1,2, \ldots, d\}$ and the short rate $r$ the market prices for risk $\theta^{k}, k \in\{1,2, \ldots, d\}$, are the only quantities that need to be specified. We assume that these processes have such integrability properties that the stochastic integrals and conditional expectations that we will form are finite.

Note that not all values for the market prices for event risk can be permitted. In the next section it will become clear that certain arbitrage opportunities arise for those market prices for event risk that lead to an infinite growth of some portfolios. This kind of arbitrage will be excluded by the following condition.

Assumption 2.4 We assume that

$$
\sqrt{h_{t}^{k}}>\theta^{k}(t)
$$

for all $t \in[0, T]$ and $k \in\{m+1, \ldots, d\}$.

As we will see below, this assumption allows us to identify the growth optimal portfolio (GOP), which will become the benchmark or numeraire for our model.

\subsection{Growth Optimal Portfolio}

For a given strictly positive portfolio process $S^{(\delta)}$ let $\pi_{\delta}^{j}(t)$ denote the corresponding $j$ th proportion of its value that is invested at time $t$ in the $j$ th primary security account. This proportion is defined by the relation

$$
\pi_{\delta}^{j}(t)=\delta^{(j)}(t) \frac{S^{(j)}(t)}{S^{(\delta)}(t)}
$$

for $t \in[0, T]$ and $j \in\{0,1, \ldots, d\}$. Furthermore, by (2.6) the proportions always add to one, that is

$$
\sum_{j=0}^{d} \pi_{\delta}^{j}(t)=1
$$


for $t \in[0, T]$. Using the vector of proportions $\pi_{\delta}(t)=\left(\pi_{\delta}^{1}(t), \ldots, \pi_{\delta}^{d}(t)\right)^{\top}$, see (2.13), we obtain for the portfolio process $S^{(\delta)}(t)$ with $(2.7),(2.11),(2.5)$ and (2.13) the SDE

$$
d S^{(\delta)}(t)=S^{(\delta)}(t-)\left(r(t) d t+\sum_{k=1}^{d} \beta_{\delta}^{k}(t-)\left(\theta^{k}(t) d t+d W^{k}(t)\right)\right)
$$

with $k$ th portfolio volatility

$$
\beta_{\delta}^{k}(t)=\sum_{j=1}^{d} \pi_{\delta}^{j}(t) b^{j, k}(t)
$$

for $k \in\{1,2, \ldots, d\}$ and $t \in[0, T]$.

Note from (2.15) and (2.2) that the process $S^{(\delta)}(t)$ with $S^{(\delta)}(0)>0$ remains almost surely strictly positive after a jump if and only if

$$
\frac{\beta_{\delta}^{k}(t-)}{\sqrt{h_{t}^{k}}}>-1
$$

for all $k \in\{m+1, \ldots, d\}$ and $t \in[0, T]$. By application of the Itô formula it follows that the logarithm of a strictly positive portfolio $S^{(\delta)}(t)$, with portfolio volatility $\beta_{\delta}^{k}$ satisfying (2.17), is governed by the SDE

$$
\begin{aligned}
d \log \left(S^{(\delta)}(t)\right)= & g_{\delta}(t) d t+\sum_{k=1}^{m} \beta_{\delta}^{k}(t) d W^{k}(t) \\
& +\sum_{k=m+1}^{d} \log \left(1+\frac{\beta_{\delta}^{k}(t-)}{\sqrt{h_{t}^{k}}}\right) \sqrt{h_{t}^{k}} d W^{k}(t)
\end{aligned}
$$

with growth rate

$$
\begin{aligned}
g_{\delta}(t)= & r(t)+\sum_{k=1}^{m}\left(\beta_{\delta}^{k}(t) \theta^{k}(t)-\frac{1}{2}\left(\beta_{\delta}^{k}(t)\right)^{2}\right) \\
& +\sum_{k=m+1}^{d}\left(\beta_{\delta}^{k}(t)\left(\theta^{k}(t)-\sqrt{h_{t}^{k}}\right)+\log \left(1+\frac{\beta_{\delta}^{k}(t)}{\sqrt{h_{t}^{k}}}\right) h_{t}^{k}\right)
\end{aligned}
$$

for $t \in[0, T]$. From the first term in the last sum on the right-hand side of equation (2.19) it follows that for all nonnegative portfolios the growth rates are bounded by a predictable process if Assumption 2.4 is satisfied.

We can now search for a GOP, which is a portfolio with maximum growth rate, as described in the following definition. 
Definition 2.5 A GOP is a strictly positive portfolio process $S^{(\underline{\underline{\delta}})}=\left\{S^{(\underline{\underline{\delta}})}(t)\right.$, $t \in[0, T]\}$ with

$$
S^{(\underline{\delta})}(0)=1
$$

and maximum growth rate $g_{\underline{\delta}}(t)$ such that

$$
g_{\underline{\delta}}(t) \geq g_{\delta}(t)
$$

for all $t \in[0, T]$ and strictly positive portfolio processes $S^{(\delta)}$.

There is an increasing literature on the GOP. We refer to Kelly (1956), Long (1990), Karatzas \& Shreve (1998) and Goll \& Kallsen (2003) for more information on this topic. As a consequence of Assumption 2.4 the market price for the $k$ th event risk $\theta^{k}(t)$ at any time $t \in[0, T]$ cannot be equal to or larger than the square root $\sqrt{h_{t}^{k}}$ of the $k$ th jump intensity for all $k \in\{m+1, m+2, \ldots, d\}$. This allows us to introduce a particular portfolio $S^{\left(\delta_{*}\right)}$ with proportions of the form

$$
\pi_{\delta_{*}}(t)=\left(\pi_{\delta_{*}}^{1}(t), \ldots, \pi_{\delta_{*}}^{d}(t)\right)^{\top}=\left(\beta_{\delta_{*}}(t)^{\top} b^{-1}(t)\right)^{\top}
$$

and $k$ th portfolio volatility

$$
\beta_{\delta_{*}}^{k}(t)=\left\{\begin{array}{cll}
\theta^{k}(t) & \text { for } \quad k \in\{1,2, \ldots, m\} \\
\frac{\theta^{k}(t)}{1-\frac{\theta^{k}(t)}{\sqrt{h_{t}^{k}}}} & \text { for } \quad k \in\{m+1, \ldots, d\}
\end{array}\right.
$$

for $t \in[0, T]$. Note that the form of the portfolio volatility $\beta_{\delta_{*}}^{k}(t), k \in\{m+$ $1, \ldots, d\}$ for the $k$ th event risk is asymptotically the same as would apply for continuous uncertainty if the corresponding jump intensity $h_{t}^{k}$ tends to infinity. Because of Assumption 2.4 relation (2.17) is for $S^{\left(\delta_{*}\right)}$ satisfied and the portfolio is therefore almost surely strictly positive.

By $(2.15)$ and (2.23) it follows that the portfolio value $S^{\left(\delta_{*}\right)}(t)$ satisfies the SDE

$$
\begin{array}{r}
d S^{\left(\delta_{*}\right)}(t)=S^{\left(\delta_{*}\right)}(t-)\left(r(t) d t+\sum_{k=1}^{m} \theta^{k}(t)\left(\theta^{k}(t) d t+d W^{k}(t)\right)\right. \\
\left.+\sum_{k=m+1}^{d} \frac{\theta^{k}(t)}{1-\frac{\theta^{k}(t)}{\sqrt{h_{t}^{k}}}}\left(\theta^{k}(t) d t+d W^{k}(t)\right)\right)
\end{array}
$$

for $t \in[0, T]$, where we set

$$
S^{\left(\delta_{*}\right)}(0)=1
$$

We can now prove the following result.

Corollary 2.6 There exists a unique GOP given by the portfolio $S^{\left(\delta_{*}\right)}$ that satisfies the SDE (2.24). 
Proof: Under Assumption 2.4 it follows by the first order conditions for identifying the maximum of the growth rate (2.19) that the optimal portfolio volatilities $\beta_{\delta_{*}}^{k}(t)$ are given by (2.23). Consequently, by (2.16) the optimal proportions $\pi_{\underline{\delta}}^{1}(t), \ldots, \pi_{\underline{\delta}}^{d}(t)$ need to solve the system of linear equations

$$
\sum_{\ell=1}^{d} \pi_{\underline{\delta}}^{\ell}(t) b^{\ell, k}(t)=\beta_{\delta_{*}}^{k}(t)
$$

for all $k \in\{1,2, \ldots, d\}$. Due to the invertibility of the generalized volatility matrix $b(t)$, see Assumption 2.3, the optimal proportions $\pi_{\underline{\delta}}(t)=\pi_{\delta_{*}}(t)$ are uniquely determined and so is the GOP. The SDE (2.24) is then by (2.15), (2.22), (2.23) and (2.16) the governing equation for the GOP.

\subsection{Benchmarked Portfolio Processes}

Let us now use the GOP $S^{\left(\delta_{*}\right)}$ as benchmark or numeraire and call the above model a benchmark model. Furthermore, values, when expressed in units of $S^{\left(\delta_{*}\right)}$, are called benchmarked values. Thus, we can consider for any portfolio $S^{(\delta)}$ its benchmarked value

$$
\hat{S}^{(\delta)}(t)=\frac{S^{(\delta)}(t)}{S^{\left(\delta_{*}\right)}(t)}
$$

at time $t \in[0, T]$. We then obtain the following result.

Corollary 2.7 Any benchmarked portfolio process $\hat{S}^{(\delta)}=\left\{\hat{S}^{(\delta)}(t), t \in[0, T]\right\}$ is an $(\underline{\mathcal{A}}, P)$-local martingale.

Proof: By application of the Itô formula together with (2.15) and (2.24) we obtain the SDE

$$
\begin{aligned}
d \hat{S}^{(\delta)}(t)= & \hat{S}^{(\delta)}(t-)\left\{\sum_{k=1}^{m}\left(\beta_{\delta}^{k}(t)-\theta^{k}(t)\right) d W^{k}(t)\right. \\
& \left.+\sum_{k=m+1}^{d}\left(\beta_{\delta}^{k}(t-)\left(1-\frac{\theta^{k}(t)}{\sqrt{h_{t}^{k}}}\right)-\theta^{k}(t)\right) d W^{k}(t)\right\}
\end{aligned}
$$

for $t \in[0, T]$. Since this SDE is driftless it follows that $\hat{S}^{(\delta)}$ is an $(\underline{\mathcal{A}}, P)$-local martingale, see Protter (1990).

Corollary 2.7 shows that any appropriately stopped benchmarked portfolio process is a martingale. This means that all benchmarked price processes behave locally in time in some sense like martingales. In our incomplete benchmark model the market prices for risk can be quite general predictable processes that 
need only to satisfy Assumption 2.4 and natural integrability conditions. Therefore, they can capture changing demand and supply conditions, adjustments to an agents's utility function or the choice of an equivalent martingale measure.

Due to a result in Ansel \& Stricker (1994), a nonnegative (negative), local martingale is a super-(sub-)martingale. This leads to the following result.

Corollary 2.8 Any benchmarked, nonnegative (negative) portfolio process $\hat{S}^{(\delta)}$ is an $(\underline{\mathcal{A}}, P)$-super-(-sub-)martingale, that is

$$
\hat{S}^{(\delta)}(t) \stackrel{(\leq)}{\geq} E\left(\hat{S}^{(\delta)}(\tau) \mid \mathcal{A}_{t}\right)
$$

for all $\tau \in[0, T]$ and $t \in[0, \tau]$.

Corollary 2.8 shows that there is no nonnegative benchmarked portfolio which generates unbounded expected returns. This appears to be a realistic and desirable property for a benchmark model.

\subsection{Basic Arbitrage}

The above arguments indicate that basic arbitrage is excluded in any benchmark model, as is confirmed by the following theorem.

Theorem 2.9 There exists no basic arbitrage in the sense of Definition 2.2.

Proof: By Corollary 2.8 any nonnegative benchmarked portfolio process $\hat{S}^{(\delta)}$ is an $(\underline{\mathcal{A}}, P)$-supermartingale. For a nonnegative benchmarked portfolio $\hat{S}^{(\delta)}$ it follows from its supermartingale property and the optional stopping theorem, see Protter (1990), that

$$
E\left(\hat{S}^{(\delta)}(\sigma) \mid \mathcal{A}_{\tau}\right) \leq \hat{S}^{(\delta)}(\tau)=0
$$

for all stopping times $\tau \in[0, T]$ and $\sigma \in[\tau, T]$. Consequently, if $S^{(\delta)}(\tau)=0$, then there is zero probability that $S^{(\delta)}(\sigma)$ is strictly positive, that is

$$
P\left(S^{(\delta)}(\sigma)>0 \mid \mathcal{A}_{\tau}\right)=P\left(\hat{S}^{(\delta)}(\sigma)>0 \mid \mathcal{A}_{\tau}\right)=0 .
$$

This shows that basic arbitrage, as described in Definition 2.2, does not exist in a benchmark model. 


\section{Pricing and Hedging}

\subsection{Fair Contingent Claim Valuation}

Let us now consider the pricing and hedging of contingent claims for the given benchmark model.

Definition 3.1 We call an $\mathcal{A}_{\tau}$-measurable payoff $H_{\tau}$, which matures at a stopping time $\tau \in[0, T]$, a contingent claim if

$$
E\left(\frac{\left|H_{\tau}\right|}{S^{\left(\delta_{*}\right)}(\tau)} \mid \mathcal{A}_{t}\right)<\infty
$$

for $t \in[0, \tau]$.

Obviously, by Corollary 2.8 the value of any portfolio $S^{(\delta)}(\tau)$ satisfies condition (3.1) and is thus a contingent claim. Furthermore, values of contingent claims at earlier times are usually called derivative prices. These exist in many forms in real markets. Besides standard traded derivative instruments they can be insurance contracts, real options and over-the-counter agreements. As we will see later on, the following interpretation of what constitutes a fair value appears to be natural.

Definition 3.2 A value process whose benchmarked values form an $(\underline{\mathcal{A}}, P)$ martingale is called fair.

Note that for a contingent claim $H_{\tau}$ the benchmarked conditional expectation $\hat{U}_{H_{\tau}}=\left\{\hat{U}_{H_{\tau}}(t), t \in[0, \tau]\right\}$ with

$$
\hat{U}_{H_{\tau}}(t)=E\left(\frac{H_{\tau}}{S^{\left(\delta_{*}\right)}(\tau)} \mid \mathcal{A}_{t}\right)
$$

forms an $(\underline{\mathcal{A}}, P)$-martingale. Therefore the corresponding value process $U_{H_{\tau}}=$ $\left\{U_{H_{\tau}}(t), t \in[0, \tau]\right\}$ is by Definition 3.2 fair, where

$$
U_{H_{\tau}}(t)=\hat{U}_{H_{\tau}}(t) S^{\left(\delta_{*}\right)}(t)
$$

for $t \in[0, \tau]$.

By using (3.2) and (3.3) the fair value $U_{H_{\tau}}(t)$ at time $t$ of a given contingent claim $H_{\tau}$ is uniquely determined by the fair pricing formula

$$
U_{H_{\tau}}(t)=E\left(\frac{S^{\left(\delta_{*}\right)}(t)}{S^{\left(\delta_{*}\right)}(\tau)} H_{\tau} \mid \mathcal{A}_{t}\right)
$$

for all $t \in[0, \tau]$. Note that the expectation in (3.4) is taken under the real world probability measure $P$. We remark that other notions of "fair" prices have been suggested in the literature, for instance, in Davis (1997) or Karatzas \& Shreve (1998). These are typically linked to the existence of an equivalent risk neutral measure. As mentioned above, this assumption is not required under the proposed benchmark approach. 


\subsection{Risk Neutral Pricing}

In the case when an equivalent risk neutral martingale measure $\tilde{P}$ exists, then it is characterized by the Radon-Nikodym derivative process $\Lambda=\{\Lambda(t), t \in[0, T]\}$ with

$$
\Lambda(t)=\frac{S^{\left(\delta_{*}\right)}(0)}{S^{\left(\delta_{*}\right)}(t)} \frac{S^{(0)}(t)}{S^{(0)}(0)}=\frac{\hat{S}^{(0)}(t)}{\hat{S}^{(0)}(0)}=\left.\frac{d \tilde{P}}{d P}\right|_{\mathcal{A}_{t}}
$$

for $t \in[0, T]$. We can therefore rewrite the fair pricing formula (3.4) in the form

$$
\begin{aligned}
U_{H_{\tau}}(t) & =E\left(\frac{\Lambda(\tau)}{\Lambda(t)} \frac{S^{(0)}(t)}{S^{(0)}(\tau)} H_{\tau} \mid \mathcal{A}_{t}\right) \\
& =\tilde{E}\left(\frac{S^{(0)}(t)}{S^{(0)}(\tau)} H_{\tau} \mid \mathcal{A}_{t}\right)
\end{aligned}
$$

for all $t \in[0, \tau]$. Here $\tilde{E}$ denotes the expectation with respect to the equivalent risk neutral martingale measure $\tilde{P}$. Note that relation (3.6) is the standard risk neutral pricing formula.

In a benchmark model we may not have an equivalent risk neutral martingale measure $\tilde{P}$ and the risk neutral pricing formula (3.6) breaks down. This is, for instance, the case when the Radon-Nikodym derivative process $\Lambda$ forms a strict local martingale. For examples of this kind we refer to Heath \& Platen (2002a, 2002b, 2002c, 2003).

\section{$3.3 \quad$ Hedging}

We say that a portfolio $S^{(\delta)}$ replicates a contingent claim $H_{\tau}$ if

$$
S^{(\delta)}(\tau)=H_{\tau}
$$

almost surely. As pointed out in Heath \& Platen (2002a, 2002b, 2002c), there may exist several self-financing portfolios in a benchmark model that replicate a given contingent claim. By Corollary 2.7 benchmarked portfolio values form local martingales. In the case of a nonnegative (negative) replicating portfolio it follows from Corollary 2.8 that its benchmarked value is a super- (sub-)martingale. A martingale that coincides at some future date with a super-(sub-)martingale cannot be larger (smaller) than the super-(sub-)martingale at any earlier date. This leads to the following conclusion.

Corollary 3.3 For a nonnegative (negative) contingent claim $H_{\tau}$ the fair portfolio $S^{\left(\delta_{H_{\tau}}\right)}$ is the minimal (maximal) portfolio that replicates the contingent claim. 
The distinguishing feature between a local martingale and a martingale is essentially an integrability property, see Protter (1990). Since local martingales behave locally in time in some sense like martingales, from a practical perspective, for short dated securities and realistic model parameters any reasonable pricing rule must be close to fair pricing.

As shown by Föllmer \& Schweizer (1991) under the assumption on the existence of an equivalent risk neutral martingale measure $\tilde{P}$, the hedging of a contingent claim is naturally linked to the existence of a corresponding martingale representation under $\tilde{P}$ for the discounted contingent claim. In a benchmark model we can use similar martingale representations. These are formulated under the real world probability measure $P$ and do not require the existence of an equivalent risk neutral measure. For functionals of Brownian motions martingale representations are described, for instance, in Karatzas \& Shreve (1991) and for Markovian semimartingale models in Jacod, Méléard \& Protter (2000). Moreover, one can directly derive martingale representations for Markovian multi-factor benchmark models by using the Feynman-Kac formula. The following assumption allows us to formulate general results without specifying a particular dynamics for the primary security accounts.

Assumption 3.4 Assume that for each contingent claim $H_{\tau}$ there exists a martingale representation for its benchmarked value of the form

$$
\frac{H_{\tau}}{S^{\left(\delta_{*}\right)}(\tau)}=\hat{U}_{H_{\tau}}(t)+\sum_{k=1}^{d} \int_{t}^{\tau} x_{H_{\tau}}^{k}(s) d W^{k}(s)
$$

for all $t \in[0, \tau]$ with a unique, predictable vector process $x_{H_{\tau}}=\left\{x_{H_{\tau}}(t)=\right.$ $\left.\left(x_{H_{\tau}}^{1}(t), \ldots, x_{H_{\tau}}^{d}(t)\right)^{\top}, t \in[0, \tau]\right\}$, where

$$
\int_{0}^{\tau} \sum_{k=1}^{d}\left(x_{H_{\tau}}^{k}(s)\right)^{2} d s<\infty
$$

almost surely.

We then prove the following result.

Theorem 3.5 For each contingent claim $H_{\tau}$ there exists a fair, replicating portfolio $S^{\left(\delta_{H_{\tau}}\right)}$, which has at time the value

$$
S^{\left(\delta_{H_{\tau}}\right)}(t)=\hat{U}_{H_{\tau}}(t) S^{\left(\delta_{*}\right)}(t),
$$

see (3.2), and is determined by the vector of proportions

$$
\pi_{\delta_{H_{\tau}}}(t)=\left(\beta_{\delta_{H_{\tau}}}(t)^{\top} b^{-1}(t)\right)^{\top}
$$


Here the vector $\beta_{\delta_{H_{\tau}}}(t)=\left(\beta_{\delta_{H_{\tau}}}^{1}(t), \ldots, \beta_{\delta_{H_{\tau}}}^{d}(t)\right)^{\top}$ has components

$$
\beta_{\delta_{H_{\tau}}}^{k}(t)=\left\{\begin{array}{cll}
\frac{x_{H_{\tau}}^{k}(t)}{\hat{U}_{H_{\tau}}(t)}+\theta^{k}(t) & \text { for } & k \in\{1,2, \ldots, m\} \\
\frac{\sqrt{h_{t}^{k}}\left(\frac{x_{H_{\tau}}^{k}(t)}{\hat{U}_{H_{\tau}}(t-)}+\theta^{k}(t)\right)}{\sqrt{h_{t}^{k}}-\theta^{k}(t)} & \text { for } & k \in\{m+1, \ldots, d\}
\end{array}\right.
$$

for $t \in[0, \tau]$.

Proof: For a given contingent claim $H_{\tau}$ we use the martingale representation (3.8). This leads us for a benchmarked hedging portfolio $\hat{S}^{\left(\delta_{H_{\tau}}\right)}$, see (2.28), to the replication condition

$$
\begin{aligned}
\frac{H_{\tau}}{S^{\left(\delta_{*}\right)}(\tau)}-\hat{U}_{H_{\tau}}(t)= & \sum_{k=1}^{d} \int_{t}^{\tau} x_{H_{\tau}}^{k}(s) d W^{k}(s) \\
= & \sum_{k=1}^{m} \int_{t}^{\tau} \hat{S}^{\left(\delta_{H_{\tau}}\right)}(s)\left(\beta_{\delta_{H}}^{k}(s)-\theta^{k}(s)\right) d W^{k}(s) \\
& +\sum_{k=m+1}^{d} \int_{t}^{\tau} \hat{S}^{\left(\delta_{H_{\tau}}\right)}(s-)\left(\beta_{\delta_{H}}^{k}(s-)\left(1-\frac{\theta^{k}(s)}{\sqrt{h_{s}^{k}}}\right)-\theta^{k}(s)\right) d W^{k}(s) \\
= & \hat{S}^{\left(\delta_{H_{\tau}}\right)}(\tau)-\hat{S}^{\left(\delta_{H_{\tau}}\right)}(t)
\end{aligned}
$$

for $t \in[0, \tau]$. The formulas (2.16) and (3.12) provide by direct comparison of the integrands in (3.13) the equation

$$
\left(\pi_{\delta_{H_{\tau}}}^{\top}(t) b(t)\right)^{\top}=\beta_{\delta_{H_{\tau}}}(t)
$$

for $t \in[0, \tau]$. By the invertibility of $b(t)$, see Assumption 2.3, this proves (3.11), and thus with (3.7) equation (3.10).

\subsection{Fluctuation Minimization Hedge}

In general, not all primary security accounts are tradable. We fix $Q \subseteq\{1,2, \ldots, d\}$ as the set of indices that characterize the traded sources of uncertainty. The set $\mathcal{C}$ denotes then the corresponding set of tradable portfolios $S^{(\delta)}$ that can be obtained by combining primary security accounts. By the SDE (2.15) these portfolios have zero $k$ th volatilities $\beta_{\delta}^{k}$ for the indices $k \in\{1,2, \ldots, d\} \backslash Q$ of the martingales $W^{k}$ which do not appear in the SDEs of the traded primary security accounts. Consequently, by (2.3) and (2.7), for all tradable portfolios $S^{(\delta)}$ the corresponding $k$ th portfolio volatility

$$
\beta_{\delta}^{k}(t)=0
$$


vanishes for all $t \in[0, T]$ when $k \in\{1,2, \ldots, d\} \backslash Q$.

A benchmark model is called complete if all contingent claims can be replicated by a tradable portfolio. Otherwise, the model is called incomplete. It is obvious that if $Q$ does not equal the set $\{1,2, \ldots, d\}$ of all indices, this means that not all sources of uncertainty are traded, then the benchmark model is incomplete.

Consider now a nonnegative contingent claim $H_{\tau}$. According to (3.10) its fair price process is given by $S^{\left(\delta_{H_{\tau}}\right)}$. We know by Corollary 3.3 that this price process equals the minimal replicating portfolio in the complete market case. However, in the given incomplete market situation $S^{\left(\delta_{H_{\tau}}\right)}$ may not be a tradable portfolio.

Let us denote by $S^{\left(\bar{\delta}_{H_{\tau}}\right)} \in \mathcal{C}$ a tradable portfolio that a hedger may use to hedge the uncertainty related to $H_{\tau}$. It arises then the question of how to choose the hedge portfolio $S^{\left(\bar{\delta}_{H_{\tau}}\right)}$. Of course, there are many possible strategies. However, note that the hedger observes at time $t$ the profit and loss (P\&L)

$$
C_{H_{\tau}, \bar{\delta}_{H_{\tau}}}(t)=S^{\left(\bar{\delta}_{H_{\tau}}\right)}(t)-S^{\left(\delta_{H_{\tau}}\right)}(t)
$$

for $t \in[0, T]$ as the difference between the hedge portfolio and the fair price. It seems to be unreasonable and expensive to hedge general market movements, that is changes in the benchmark. To adjust for movements in the benchmark we consider the benchmarked P\&L

$$
\hat{C}_{H_{\tau}, \bar{\delta}_{H_{\tau}}}(t)=\frac{C_{H_{\tau}, \bar{\delta}_{H_{\tau}}}(t)}{S^{\left(\delta_{*}\right)}(t)}
$$

for $t \in[0, \tau]$. The benchmarked P\&L satisfies by (3.15), (3.16) and (2.28) at time $t$ the equation

$$
\begin{aligned}
\hat{C}_{H_{\tau}, \bar{\delta}_{H_{\tau}}}(t)=\hat{C}_{H_{\tau}, \bar{\delta}_{H_{\tau}}}\left(t_{0}\right)+\sum_{k=1}^{m} \int_{t_{0}}^{t}\left[\hat{S}^{\left(\bar{\delta}_{H_{\tau}}\right)}(s)\left(\beta_{\bar{\delta}_{H_{\tau}}}^{k}(s)-\theta^{k}(s)\right)\right. \\
\left.\left.\quad-\hat{S}^{\left(\delta_{H_{\tau}}\right)}(s)\left(\beta_{\delta_{H_{\tau}}}^{k}(s)-\theta^{k}(s)\right)\right] d W^{k}(s)\right) \\
+\sum_{k=m+1}^{d} \int_{t_{0}}^{t}\left[\hat{S}^{\left(\bar{\delta}_{H_{\tau}}\right)}(s-)\left(\beta_{\bar{\delta}_{H_{\tau}}}(s-)\left(1-\frac{\theta^{k}(s)}{\sqrt{h_{s}^{k}}}\right)-\theta^{k}(s)\right)\right. \\
\left.\left.\quad-\hat{S}^{\left(\delta_{H_{\tau}}\right)}(s-)\left(\beta_{\delta_{H_{\tau}}}^{k}(s-)\left(1-\frac{\theta^{k}(s)}{\sqrt{h_{s}^{k}}}\right)-\theta^{k}(s)\right)\right] d W^{k}(s)\right)
\end{aligned}
$$

for $t_{0} \in[0, \tau]$ and $t \in\left[t_{0}, \tau\right]$.

When setting up the hedge portfolio, say, at the initial time $t_{0} \in[0, \tau]$, it is natural for the hedger to aim for vanishing expected benchmarked P\&L. Furthermore, the hedger is naturally concerned about the fluctuations of the benchmarked P\&L. To 
model these objectives we introduce a quadratic criterion, see also Heath, Platen \& Schweizer (2001a, 2001b), that minimizes the fluctuation

$$
F_{H_{\tau}, \bar{\delta}_{H_{\tau}}}\left(t_{0}, t\right)=E\left(\left(\hat{C}_{H_{\tau}, \bar{\delta}_{H_{\tau}}}(t)\right)^{2} \mid \mathcal{A}_{t_{0}}\right)
$$

for all $t \in\left[t_{0}, \tau\right]$. This means, we minimize the second moment of the benchmarked P\&L.

We obtain then from (3.15) - (3.18) for the fluctuation the expression

$$
\begin{aligned}
F_{H_{\tau}, \bar{\delta}_{H_{\tau}}}\left(t_{0}, t\right)= & \left.\hat{S}^{\left(\bar{\delta}_{H_{\tau}}\right)}\left(t_{0}\right)-\hat{S}^{\left(\delta_{H_{\tau}}\right)}\left(t_{0}\right)\right)^{2} \\
+ & \int_{t_{0}}^{t}\left\{\sum _ { k = 1 } ^ { m } E \left(\left[\hat{S}^{\left(\bar{\delta}_{H_{\tau}}\right)}(s)\left(\beta_{\bar{\delta}_{H_{\tau}}}^{k}(s)-\theta^{k}(s)\right)\right.\right.\right. \\
& \left.\left.-\hat{S}^{\left(\delta_{H_{\tau}}\right)}(s)\left(\beta_{\delta_{H_{\tau}}}^{k}(s)-\theta^{k}(s)\right)\right]^{2} \mid \mathcal{A}_{t_{0}}\right) \\
+ & \sum_{k=m+1}^{d} E\left(\left[\hat{S}^{\left(\bar{\delta}_{H_{\tau}}\right)}(s-)\left(\beta_{\bar{\delta}_{H_{\tau}}}^{k}(s-)\left(1-\frac{\theta^{k}(s)}{\sqrt{h_{s}^{k}}}\right)-\theta^{k}(s)\right)\right.\right. \\
& \left.\left.\left.-\hat{S}^{\left(\delta_{H_{\tau}}\right)}(s-)\left(\beta_{\delta_{H_{\tau}}}^{k}(s-)\left(1-\frac{\theta^{k}(s)}{\sqrt{h_{s}^{k}}}\right)-\theta^{k}(s)\right)\right]^{2} \mid \mathcal{A}_{t_{0}}\right)\right\} d s
\end{aligned}
$$

for $t \in\left[t_{0}, \tau\right]$. To minimize the fluctuation (3.19) the hedger can exclude the terms that are due to traded uncertainty by choosing the generalized portfolio volatility

$$
\beta_{\bar{\delta}_{H_{\tau}}}^{k}(t)=\frac{S^{\left(\delta_{H_{\tau}}\right)}(t)}{S^{\left(\bar{\delta}_{H_{\tau}}\right)}(t)}\left(\beta_{\delta_{H_{\tau}}}^{k}(t)-\theta^{k}(t)\right)+\theta^{k}(t)
$$

for all $k \in Q \cap\{1,2, \ldots, m\}$ and

$$
\beta_{\bar{\delta}_{H_{\tau}}}^{k}(t)=\left[\frac{S^{\left(\delta_{H_{\tau}}\right)}(t)}{S^{\left(\bar{\delta}_{H_{\tau}}\right)}(t)}\left(\beta_{\delta_{H_{\tau}}}^{k}(t)\left(1-\frac{\theta^{k}(s)}{\sqrt{h_{s}^{k}}}\right)-\theta^{k}(t)\right)+\theta^{k}(t)\right]\left(1-\frac{\theta^{k}(s)}{\sqrt{h_{s}^{k}}}\right)^{-1}
$$

for $k \in Q \cap\{m+1, \ldots, d\}$ and $t \in[0, \tau]$. Since by (3.14) we have

$$
\beta \frac{k}{\bar{\delta}_{H_{\tau}}}(t)=0
$$

for all $k \notin Q$ and $t \in[0, \tau]$ we get then from (3.17), (3.20) and (3.21) for the benchmarked P\&L the SDE

$$
\left.d \hat{C}_{H_{\tau}, \bar{\delta}_{H_{\tau}}}(t)=-\sum_{k \notin Q}\left(\hat{S}^{\left(\delta_{H_{\tau}}\right)}(t-) \beta_{\delta_{H_{\tau}}}^{k}(t-)+\theta^{k}(t) \hat{C}_{H_{\tau}, \bar{\delta}_{H_{\tau}}}(t-)\right) d W^{k}(t)\right)
$$


for $t \in[0, T]$. This shows that with the choice (3.20) - (3.21) only nontraded uncertainty remains in the benchmarked P\&L. To determine the initial condition for setting up the hedge at time $t_{0}$ it follows from the minimization of the first term of the right hand side of (3.18) that we should set

$$
\left(\hat{S}^{\left(\bar{\delta}_{H_{\tau}}\right)}\left(t_{0}\right)-\hat{S}^{\left(\delta_{H_{\tau}}\right)}\left(t_{0}\right)\right)^{2}=0
$$

By (3.24) and (2.27) the initial value is therefore the fair price

$$
S^{\left(\bar{\delta}_{H_{\tau}}\right)}\left(t_{0}\right)=S^{\left(\delta_{H_{\tau}}\right)}\left(t_{0}\right)
$$

Since the fluctuations of the benchmarked P\&L are minimized by the resulting hedging portfolio $S^{\left(\bar{\delta}_{H_{\tau}}\right)}$, we call this the fluctuation minimization hedge. It is important to note that the corresponding proportions can be directly computed from (2.16).

Corollary 3.6 For the fluctuation minimization hedge the proportions of the hedge portfolio at time $t$ satisfy the relation

$$
\pi_{\bar{\delta}_{H_{\tau}}}(t)=\left(\beta_{\bar{\delta}_{H_{\tau}}}(t)^{\top} b^{-1}(t)\right)^{\top}
$$

for $t \in[0, \tau]$, where the volatility vector $\beta_{\bar{\delta}_{H_{\tau}}}(t)=\left(\beta_{\bar{\delta}_{H_{\tau}}}^{1}(t), \ldots, \beta_{\bar{\delta}_{H_{\tau}}}^{d}(t)\right)^{\top}$ of the hedge portfolio $S^{\left(\bar{\delta}_{H_{\tau}}\right)}$ is given by (3.20) - (3.22) and the initial value for the hedge portfolio equals the fair price (3.25).

Obviously, in the case when the market is complete, then one obtains the same hedge portfolio as described in Theorem 3.5 and the fluctuation is zero. In the incomplete market case the benchmarked P\&L is an $(\underline{\mathcal{A}}, P)$-martingale. Therefore, its actual value is the best forecast of the terminal benchmarked P\&L. This hedging approach operates relative to the benchmark and appears to be natural and constructive. It simply removes from the benchmarked P\&L the tradable part. Furthermore, the P\&L tends to zero as the market is completed. The fair price of the contingent claim arises as the natural initial price for setting up a fluctuation minimization hedge. Since the benchmark is the GOP it can be interpreted as the best performing portfolio. Under fluctuation minimization the deviations of the P\&L from zero are minimized relative to this benchmark.

\section{Acknowledgement}

The author would like to thank Morten Christensen, Nicole El Karoui, Robert Elliott, David Heath, Marek Musiela, Chris Rogers, Wolfgang Runggaldier, Wolfgang Schmidt, Albert Shiryaev and Thaleia Zariphopoulou for their interest in this research and stimulating discussions on the subject. 


\section{References}

Ansel, J. P. \& C. Stricker (1994). Couverture des actifs contingents. Ann. Inst. H. Poincaré Probab. Statist. 30, 303-315.

Bajeux-Besnainou, I. \& R. Portait (1997). The numeraire portfolio: A new perspective on financial theory. The European Journal of Finance 3, 291309.

Davis, M. H. A. (1997). Option pricing in incomplete markets. In M. A. H. Dempster and S. R. Pliska (Eds.), Mathematics of derivative securities, pp. 227-254. Cambridge University Press.

Davis, M. H. A. (2003). Valuation, hedging and investing in incomplete financial markets. In Proceedings ICIAM03 World Congress, Sydney, June 2003. (to appear).

Delbaen, F. \& W. Schachermayer (1995). The no-arbitrage property under a change of numeraire. Stochastics Stochastics Rep. 53, 213-226.

Delbaen, F. \& W. Schachermayer (1998). The fundamental theorem of asset pricing for unbounded stochastic processes. Math. Ann 312, 215-250.

El Karoui, N. (2003). paper presented at Snowbird. In Proceedings AMS-SIAM Mathematical Finance Conference. to appear in this issue.

Elliott, R. J. (2003). paper presented at Snowbird. In Proceedings AMS-SIAM Mathematical Finance Conference. to appear in this issue.

Föllmer, H. \& M. Schweizer (1991). Hedging of contingent claims under incomplete information. In M. Davis and R. Elliott (Eds.), Applied Stochastic Analysis, Volume 5 of Stochastics Monogr., pp. 389-414. Gordon and Breach, London/New York.

Föllmer, H. \& D. Sondermann (1986). Hedging of non-redundant contingent claims. In W. Hildebrandt and A. Mas-Colell (Eds.), Contributions to Mathematical Economics, pp. 205-223. North Holland.

Geman, S., N. El Karoui, \& J. C. Rochet (1995). Changes of numeraire, changes of probability measures and pricing of options. J. Appl. Probab. 32, 443458.

Goll, T. \& J. Kallsen (2003). A complete explicit solution to the log-optimal portfolio problem. Adv. in Appl. Probab. 13(2), 774-799.

Heath, D. \& E. Platen (2002a). Consistent pricing and hedging for a modified constant elasticity of variance model. Quant. Finance. 2(6), 459-467.

Heath, D. \& E. Platen (2002b). Perfect hedging of index derivatives under a minimal market model. Int. J. Theor. Appl. Finance 5(7), 757-774.

Heath, D. \& E. Platen (2002c). Pricing and hedging of index derivatives under an alternative asset price model with endogenous stochastic volatility. In J. Yong (Ed.), Recent Developments in Mathematical Finance, pp. 117-126. World Scientific. 
Heath, D. \& E. Platen (2003). Pricing of index options under a minimal market model with lognormal scaling. Quant. Finance. 3(6), 442-450.

Heath, D., E. Platen, \& M. Schweizer (2001a). A comparison of two quadratic approaches to hedging in incomplete markets. Math. Finance 11(4), 385413.

Heath, D., E. Platen, \& M. Schweizer (2001b). Numerical comparison of local risk-minimisation and mean-variance hedging. In E. Jouini, J. Cvitanić, and M. Musiela (Eds.), Option Pricing, Interest Rates and Risk Management, Handbooks in Mathematical Finance, pp. 509-537. Cambridge University Press.

Hofmann, N., E. Platen, \& M. Schweizer (1992). Option pricing under incompleteness and stochastic volatility. Math. Finance 2(3), 153-187.

Jacod, J., S. Méléard, \& P. Protter (2000). Explicit form and robustness of martingale representations. Ann. Probab. 28(4), 1747-1780.

Karatzas, I. \& S. E. Shreve (1991). Brownian Motion and Stochastic Calculus (2nd ed.). Springer.

Karatzas, I. \& S. E. Shreve (1998). Methods of Mathematical Finance, Volume 39 of Appl. Math. Springer.

Kelly, J. R. (1956). A new interpretation of information rate. Bell Syst. Techn. J. 35, 917-926.

Long, J. B. (1990). The numeraire portfolio. J. Financial Economics 26, 29-69.

Musiela, M. \& T. Zariphopoulou (2003). paper presented at Snowbird. In Proceedings AMS-SIAM Mathematical Finance Conference. to appear in this issue.

Platen, E. (2001). A minimal financial market model. In Trends in Mathematics, pp. 293-301. Birkhäuser.

Platen, E. (2002). Arbitrage in continuous complete markets. Adv. in Appl. Probab. 34(3), 540 - 558.

Platen, E. (2004). A class of complete benchmark models with intensity based jumps. J. Appl. Probab. 41(1). (to appear in March 2004).

Protter, P. (1990). Stochastic Integration and Differential Equations. Springer. 


\section{CONTEMPORARY MATHEMATICS}

\section{1}

\section{Mathematics of Finance}

\section{Proceedings of an AMS-IMS-SIAM}

Joint Summer Research Conference

on Mathematics of Finance June 22-26, 2003

Snowbird, Utah

George Yin

Qing Zhang

Editors 


\section{Contents}

Preface ix

List of Speakers and Title of Talks

Credit Barrier Models in a Discrete Framework 1 Claudio Albanese and Oliver X. Chen

Optimal Derivatives Design under Dynamic Risk Measures 13 Pauline Barrieu and Nicole El Karoui

On Pricing of Forward and Futures Contracts on Zero-Coupon Bonds in the Cox-Ingersoll-Ross Model JģDRZEJ BIALKOWSKI and JACEK JAKUBOwSKI

Pricing and Hedging of Credit Risk: Replication and Mean-Variance Approaches (I)

Tomasz R. Bielecki, Monique Jeanblanc, and Marek Rutkowski

Pricing and Hedging of Credit Risk: Replication and Mean-Variance Approaches (II)

Tomasz R. Bielecki, Monique Jeanblanc, and Marek Rutkowski

Spot Convenience Yield Models for the Energy Markets RENÉ Carmona and Michael LUdKovski

Optimal Portfolio Management with Consumption

Netzahualcóyotl Castañeda-Leyva and DANIEL HERnÁNDEZ-HERnÁNDEZ

Some Processes Associated with a Fractional Brownian Motion T. E. DUNCAN

Pricing Claims on Non Tradable Assets Robert J. ElliotT and John VAN DER HOEK

Some Optimal Investment, Production and Consumption Models WENDELL H. Fleming

Asian Options under Multiscale Stochastic Volatility Jean-Pierre Fouque and Chuan-Hsiang Han

A Regime Switching Model: Statistical Estimation. Empirical Evidence, and Change Point Detection XIN Guo 
Multinomial Maximum Likelihood Estimation of Market Parameters for Stock Jump-Diffusion Models

Floyd B. Hanson, John J. Westman, and Zongwu Zhu

Optimal Terminal Wealth under Partial Information

for HMM Stock Returns

ULRICH G. HAUSSMANN and JöRN SASS

Computing Optimal Selling Rules for Stocks Using Linear Programming KuRT HeLMes

Optimization of Consumption and Portfolio and Minimization of Volatility YAOZHONG HU

Options: To Buy or not to Buy?

Mattias Jonsson and RonniE Sircar

Risk Sensitive Optimal Investment: Solutions of the Dynamical Programming Equation

H. KAISE and S. J. SheU

Hedging Default Risk in an Incomplete Market ANDREW E.B. LIM

Mean-Variance Portfolio Choice with Discontinuous Asset Prices and

Nonnegative Wealth Processes ANDREW E.B. LIM and XUN YU ZHOU

Indifference Prices of Early Exercise Claims Marek Musiela and Thaleia Zariphopoulou

Random Walk around Some Problems in Identification and Stochastic

Adaptive Control with Applications to Finance Bozenna Pasik-Duncan

Pricing and Hedging for Incomplete Jump Diffusion Benchmark Models ECKHARD Platen

Why is the Effect of Proportional Transaction Costs $O\left(\delta^{2 / 3}\right)$ ?

L.C.G. ROGERS

Estimation via Stochastic Filtering in Financial Market Models WOLFGANG J. RUNGGALDIER

Stochastic Optimal Control Modeling of Debt Crises Jerome L. Stein

Duality and Risk Sensitive Portfolio Optimization LuKasz STETTNER

Characterizing Option Prices by Linear Programs RichaRd H. STOCKBRIDGE

Pricing Defaultable Bond with Regime Switching J.W. WANG and Q. ZHANG 
Affine Regime-Switching Models for Interest Rate Term Structure SHU WU and YoNG ZENG

Stochastic Approximation Methods for Some Finance Problems G. YIN and Q. ZHANG 